J. Lake Sci. (湖泊科学), 2014, 26(6): 887-896

http://www. jlakes. org. E-mail : jlakes@niglas.ac.cn

(c) 2014 by Journal of Lake Sciences

\title{
巢湖悬浮物中有机氯农药的分布、来源与风险”
}

\author{
王卿梅,何玘霜,王 雁,何 伟,秦 宁, 孔祥殝,刘文秀,徐福留** \\ (北京大学城市与环境学院地表过程分析与模拟教育部重点实验室,北京 100871)
}

\begin{abstract}
摘 要: 利用 GC-MS 测定了 2010 年 5 月至 2011 年 4 月巢湖 4 个采样点悬浮物中有机氯农药 (OCPs) 含量, 分析了悬浮物 中 OCPs 残留水平的时空变化及其组成特征, 探讨了悬浮物中主要 OCPs 的可能来源与生态风险. 结果表明: (1) 巢湖悬 浮物中共检出 17 种 $\mathrm{OCPs}$, 分别为六六六类 $(\mathrm{HCHs})$ 农药的 4 种异构体 $(\alpha-、 \beta-、 \gamma-\delta-\mathrm{HCH})$ 、滴滴涕类 (DDTs) 农药的两种 母体 ( o , $\left.\mathrm{p}^{\prime}-\mathrm{p}, \mathrm{p}^{\prime}-\mathrm{DDT}\right)$ 和 3 种代谢产物 ( $\left.\mathrm{o}, \mathrm{p}^{\prime}-\mathrm{p}, \mathrm{p}^{\prime}-\mathrm{DDE}, \mathrm{p}, \mathrm{p}^{\prime}-\mathrm{DDD}\right)$ 、六氯苯、七氯、艾氏剂、异艾氏剂、氯丹的两种异构 体 ( $\alpha$ - 、 $\gamma$-chlordane)、硫丹-I 和灭蚁灵. (2) 巢湖悬浮物中 $\sum O C P s$ 含量为 $172.7 \pm 434.9 \mathrm{ng} / \mathrm{g}$, 其中 $\sum H C H s$ 含量为 $15.1 \pm$ $10.3 \mathrm{ng} / \mathrm{g}, \sum$ DDTs 含量为 $138.8 \pm 407.3 \mathrm{ng} / \mathrm{g}$; DDTs 在夏季污染水平达到最高;空间上, 污染程度为: 东部湖区 > 西部湖 区 > 中部湖区. (3) DDTs 在总 OCPs 中占有绝对优势, DDTs 比例夏季最高而秋季最低; HCHs 仅在秋季为主要污染物. (4) HCHs 主要来源于近期林丹的非法使用; 而 DDT 主要来源于工业 DDT 的使用,在春季可能存在 DDT 的非法使用; 悬 浮物中 DDT 的降解环境主要为有氧环境. (5) 巢湖悬浮物中 $\mathrm{p}, \mathrm{p}^{\prime}$-DDT 和 $\mathrm{o}, \mathrm{p}^{\prime}$-DDT 会对水生生物产生潜在的不利影响. 关键词: 巢湖;悬浮物;有机氯农药;残留水平;来源; 风险
\end{abstract}

\section{Distribution, source identification and risk assessment of organochlorine pesticides (OCPs) in suspended particulate matter (SPM) from Lake Chaohu}

WANG Qingmei, HE Qishuang, WANG Yan, HE Wei, QIN Ning, KONG Xiangzhen, LIU Wenxiu \& XU Fuliu

(MOE Laboratory for Earth Surface Process, College of Urban and Environmental Sciences, Peking University, Beijing 100871, P. R. China)

\begin{abstract}
The residual levels of organochlorine pesticides (OCPs) in suspended particulate matter (SPM) of four sampling sites collected from Lake Chaohu ( May 2010 - April 2011) were measured. The temporal-spatial variation and composition of OCPs were analyzed and potential sources and ecological risks of OCPs were also explored. Results showed that: (1) Seventeen types of OCPs were detected in the SPM samples of Lake Chaohu, including 4 isomers of HCHs $\left(\alpha^{-}, \beta^{-}, \gamma^{-}, \delta-\mathrm{HCH}\right)$, two parent DDT compounds (o,p'-, p, p'-DDT) and their three metabolic products (o,p'-, p, p'-DDE, p, p'-DDD), HCB, Heptachlor, Aldrin, Isodrin, 2 isomers of Chlordane ( $\alpha$-, $\gamma$-chlordane), Endosulfan I and Mirex. (2) The annual average concentration of $\sum$ OCPs in the SPM of Lake Chaohu was $172.7 \pm 434.9 \mathrm{ng} / \mathrm{g}$, and HCHs and DDTs accounted for $15.1 \pm 10.3$ and $138.8 \pm$ $407.3 \mathrm{ng} / \mathrm{g}$, respectively. The DDT content reached a maximum value in the summer. Regarding the spatial variability in the degree of pollution, the following trend was observed: eastern lake > western lake > central lake. (3) DDTs were the primary constituents of the total OCPs. The proportion of DDTs was the highest in the summer and the lowest in the fall. However, HCHs were the most significant pollutants only in the fall. (4) Considering possible sources, HCHs were derived from the illegal use of lindane, while DDTs likely originated from the industrial use of DDTs and illegal use in the spring. Moreover, DDTs degraded primarily under aerobic conditions. (5) In the SPM of Lake Chaohu, residual p,p'-DDT and o,p' -DDT may have adverse impact on the aquatic organisms.
\end{abstract}

Keywords: Lake Chaohu; SPM; OCPs; residuals; sources; risk

* 国家自然科学基金项目 (41030529, 41271462)、国家水体污染控制与治理科技重大专项项目(2012ZX07103-002)和国 家杰出青年基金项目 (40725004) 联合资助. 2013-12-30 收稿;2014-04-14 收修改稿. 王卿梅(1987 ), 女, 硕士 研究生;E-mail:wangqm1015@163. com.

** 通信作者;E-mail:xufl@ urban. pku. edu. cn. 
有机氯农药 $\left(\mathrm{OCPs}\right.$ ) 作为一类典型的持久性有机污染物 ${ }^{[1]}$, 因其高毒性、持久性、半挥发性和生物富集 性等特点 ${ }^{[2-5]}$ 在国际上引起了广泛关注. 目前, 持久性有机污染物 (POPs) 公约禁止生产的 21 种 POPs 中有 13 种为 OCPs. 虽然很多国家已经停止或减少 OCPs 的使用, 但由于其在历史时期的大量使用, 至今仍在很 多食品和环境介质中被检测出 ${ }^{[6-9]}$.

OCPs 可以通过工业排放、降水、大气的干湿沉降等方式进人水体环境 ${ }^{[5,10]}$. 悬浮物作为水体环境中一 种重要的介质, 对 OCPs 在水体环境中的分配运输影响重大. 一方面, 由于 OCPs 大多具有疏水亲脂性, 其主 要吸附于悬浮颗粒物中, 并可通过沉降作用进人沉积物, 沉积物的再悬浮作用也可再次转化为悬浮物存在, 因此悬浮物担当着源和迁移载体的重要角色 ${ }^{[11]}$. 另一方面, 悬浮相中的 OCPs 在一定条件下也会进人水 相 ${ }^{[5]}$, 不仅对水生生物造成影响, 而且还会因饮水或食物链的传递给人体健康带来危害. 但近年来国内外对 河流、湖泊等水体中 OCPs 的研究多集中于水体 ${ }^{[12-14]}$ 、沉积物 ${ }^{[15-17]}$, 而对悬浮颗粒物的研究较少 ${ }^{[5,11,18]}$.

巢湖 $\left(30^{\circ} 25^{\prime} 28^{\prime \prime} \sim 31^{\circ} 43^{\prime} 28^{\prime \prime} \mathrm{N}, 117^{\circ} 16^{\prime} 54^{\prime \prime} \sim 117^{\circ} 51^{\prime} 46^{\prime \prime} \mathrm{E}\right.$ ) 位于安徽省中部, 是中国第 5 大淡水湖泊, 水 域面积约 $760 \mathrm{~km}^{2}$. 巢湖不仅为当地渔业发展和农业灌溉提供资源, 还是周边地区 960 万居民饮用水的水源 地, 因此其污染状况不仅会危及水生生态系统的安全, 也给周边的居民带来健康风险. 目前, 针对巢湖悬浮 物 OCPs 污染状况的研究较少, 主要集中在水体、沉积物上. 本文对巢湖湖区悬浮物中 OCPs 的残留水平、时 空变化、组成 (主要是 DDTs 和 HCHs) 及来源进行研究, 分析其潜在的生态风险, 以期为巢湖水域的环境保 护和生态风险评价提供科学依据.

\section{1 材料与方法}

\section{1 样品采集与富集}

2010 年 5 月至 2011 年 4 月每月进行现场采样, 在巢湖东、中、西部湖区共布设了 4 个水体采样点 (图 1 ), 分别位于巢湖水质自动监测站 $(\mathrm{JC})$ 、中庙南 $200 \mathrm{~m}(\mathrm{ZM})$ 、湖心姥山岛东 $200 \mathrm{~m}$ ( MS) 和西部塘西原自 来水厂取水口南部 $150 \mathrm{~m}(\mathrm{TX})$. 每个样点取水 $20 \mathrm{~L}$, 混匀后取 $1 \mathrm{~L}$ 水样过 $0.7 \mu \mathrm{m}$ 玻璃纤维滤膜 $\left(450^{\circ} \mathrm{C}\right.$ 高 温灼烧 $4 \mathrm{~h}$ 后至恒重), 过滤装置为 Millipore 公司型号为 80EL005 的蠕动原连接直径为 $142 \mathrm{~mm}$ 的过滤 盘. 过滤后的滤膜自然晾干后用铝箔纸包好带回实验室恒重后精确称重. 两次重量之差即为相应悬浮物 的质量.

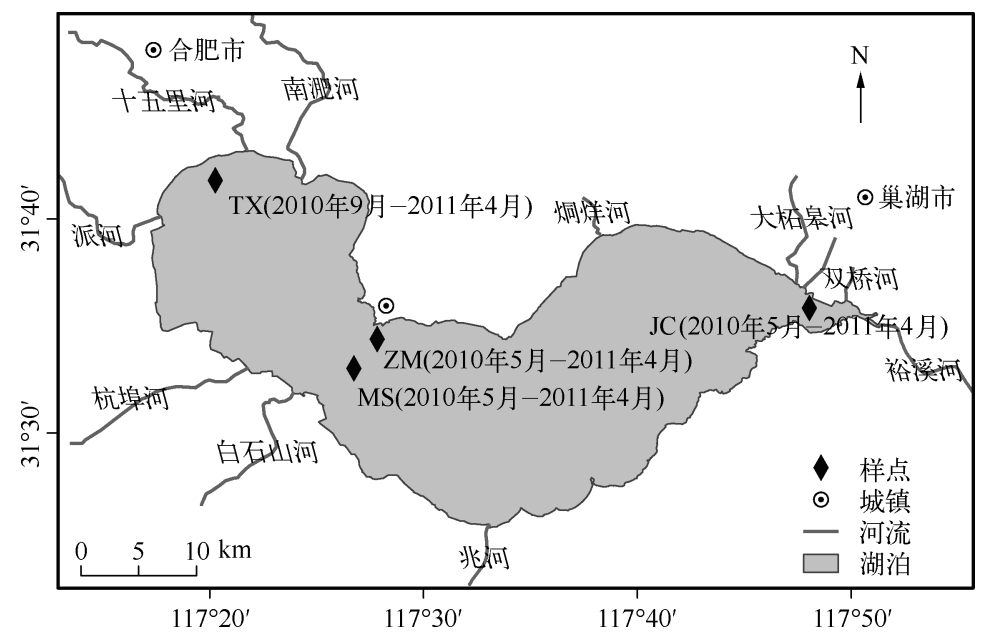

图 1 巢湖悬浮物采样点分布

Fig. 1 Distribution of sampling sites in Lake Chaohu

\section{2 样品提取与净化}

悬浮物样品使用微波萃取. 将称重后的滤膜用不锈钢剪刀剪碎, 放人微波萃取管. 向萃取管中加人 $25 \mathrm{ml}$ 正己烷与丙酮的混合溶剂 ( V:V =1:1), 再加人 $50 \mathrm{ng}$ 回收率指示物一溴二硝基苯. 用微波消解/萃取系 
统于 $1200 \mathrm{w}$ 按以下步骤提取: $10 \mathrm{~min}$ 升温至 $100^{\circ} \mathrm{C}$, 保持 $10 \mathrm{~min}$, 降温 $30 \mathrm{~min}$. 萃取液经压滤后, 装人茄形 瓶, 旋蒸 (R-201 旋转蒸发仪, 上海申生科技有限公司) 至 $1 \mathrm{ml}$ 后加人 $10 \mathrm{ml}$ 正已烷替换溶剂, 再旋蒸至 $1 \mathrm{ml}$ 待净化.

提取液用硅胶一中性氧化铝混合柱净化. 脱脂棉用二氯甲烷索氏提取 $20 \mathrm{~h}$ 备用; 硅胶选用 $80 \sim 200$ 目, 中性氧化铝选用 $100 \sim 200$ 目,均在 $450^{\circ} \mathrm{C}$ 下焙烧 $6 \mathrm{~h}, 180^{\circ} \mathrm{C}$ 下活化 $12 \mathrm{~h}$, 使用前 $1 \sim 2 \mathrm{~h}$ 用 $3 \%$ 的去离子水脱 活, 摇匀后加人正已烷没过硅胶/氧化铝, 静置平衡 $12 \mathrm{~h}$ 备用; 无水硫酸钠在 $450^{\circ} \mathrm{C}$ 下焙烧 $6 \mathrm{~h}$, 放人保干器备 用. 采用湿法装柱: 柱底端铺一层脱脂棉后, 加人正已烷润洗柱子, 依次装人 $12 \mathrm{~cm}$ 中性氧化铝、12 cm中性 硅胶、 $1 \mathrm{~cm}$ 无水硫酸钠. 将浓缩的提取液转移至混合柱中, 先加人 $20 \mathrm{ml}$ 正己烷洗去正构烷烃, 淋洗液弃去; 再加人 $50 \mathrm{ml}$ 正己烷与二氯甲烷的混合溶剂 $(\mathrm{V}: \mathrm{V}=1: 1)$ 洗脱目标污染物, 收集淋洗液旋蒸浓缩后替换正己 烷溶液,浓缩至约 $1 \mathrm{ml}$,加人内标五氯硝基苯,装人 GC 样品瓶待测.

\section{3 样品分析}

使用 Agilent 6890 GC-5976C 气相色谱质谱检测 器测定样品, 色谱柱为 HP-5MS 石英毛细管柱 $(30 \mathrm{~m}$ $\times 0.25 \mathrm{~mm} \times 0.25 \mathrm{~mm})$. 气相色谱载气为氦气, 流 速 $1 \mathrm{ml} / \mathrm{min}$,进样口温度 $220^{\circ} \mathrm{C}$, 不分流进样,进样量 $1 \mathrm{~L}$. 升温程序为: 初始温度 $50^{\circ} \mathrm{C}$ 保持 $2 \mathrm{~min}$, 以 $10^{\circ} \mathrm{C} /$ $\min$ 速度升至 $150^{\circ} \mathrm{C}$, 再以 $3^{\circ} \mathrm{C} / \mathrm{min}$ 速度继续升至 $240^{\circ} \mathrm{C}$ 保持 $5 \mathrm{~min}$,最后以 $10^{\circ} \mathrm{C} / \mathrm{min}$ 速度升至 $300^{\circ} \mathrm{C}$ 保持 $5 \mathrm{~min}$. 质谱离子源温度 $200^{\circ} \mathrm{C}$, 传输线温度 $250^{\circ} \mathrm{C}$, 四级杆温度 $150^{\circ} \mathrm{C}$. 以标准曲线内标法, 选择 离子模式定量化合物含量.

\section{4 质保与质控}

样品分析过程中增加质量控制/质量保证 ( QA) QC) 以控制样品分析,包括方法空白、加标空白、基质 加标、样品平行样和标准参考物质, 并用指示物控制 整个操作流程的回收率. 基于 $0.1 \mathrm{~g}$ 悬浮颗粒物计 算的悬浮物中各 OCPs 的回收率和检出限见表 1 .

\section{2 结果与讨论}

\section{1 悬浮物中 OCPs 的残留水平}

44 个巢湖悬浮物样品中共有 17 种 OCPs 检出, 分别为: $\mathrm{HCHs}$ 的 4 种异构体 $(\alpha-、 \beta-、 \gamma-\delta-\mathrm{HCH})$ 、 DDTs 类的 2 种母体及其 3 种代谢产物 ( $\mathrm{o}, \mathrm{p}^{\prime}-、 \mathrm{p}, \mathrm{p}^{\prime}$ DDT、DDE, p, p'-DDD)、六氯苯 (HCB) 、七氯 (Heptachlor)、艾氏剂 (Aldrin)、异艾氏剂（Isodrin）、氯丹
表 1 OCPs 回收率和检出限

Tab. 1 Recoveries and detection limits of OCPs

\begin{tabular}{ccc}
\hline OCPs & 回收率/\% & 检出限 $/(\mathrm{ng} / \mathrm{g})$ \\
\hline$\alpha$-HCH & 87.5 & 0.5 \\
$\beta$-HCH & 107.7 & 0.5 \\
$\gamma$-HCH & 93.1 & 0.5 \\
$\delta$-HCH & 94.1 & 0.5 \\
o,p'-DDE & 112.0 & 1 \\
$\mathrm{p}, \mathrm{p}^{\prime}$-DDE & 117.7 & 0.5 \\
o,p'-DDD & 114.1 & 0.5 \\
$\mathrm{p}, \mathrm{p}^{\prime}$-DDD & 84.5 & 5 \\
o,p'-DDT & 115.8 & 5 \\
$\mathrm{p}, \mathrm{p}^{\prime}$-DDT & 135.8 & 0.5 \\
HCB & 76.3 & $<0.05$ \\
Heptachlor & 110.4 & 0.5 \\
Aldrin & 96.6 & 0.5 \\
Isodrin & 95.5 & 0.2 \\
$\alpha$-chlordane & 104.2 & 1 \\
$\gamma$-chlordane & 98.7 & 0.5 \\
Endosulfan II & 98.1 & 0.5 \\
Dieldrin & 90.5 & 0.5 \\
Endrin & 142.4 & 5 \\
Endosulfan I & 84.7 & 0.5 \\
Mirex & 108.2 & 1 \\
Methoxychlor & 79.5 & 0.5 \\
\hline & & \\
\hline & & 5 \\
\hline
\end{tabular}

(Chlordane) 的 2 种异构体 ( $\alpha$-、 $\gamma$-chlordane) 、硫丹-I(Endosulfan I) 和灭蚁灵 (Mirex). 其中, $\alpha-\mathrm{HCH} 、 \beta$-HCH、 $\gamma$-HCH、p, $\mathrm{p}^{\prime}$-DDE 、o, $\mathrm{p}^{\prime}$-DDT、HCB 、Isodrin 的检出率为 100\%, Mirex 的检出率低于 $50 \%$, 其余 OCPs 的检出率 在 $62.5 \% \sim 95.8 \%$ 之间 (表 2).

巢湖悬浮物中 $\sum O C P s$ 年平均含量为 $172.7 \pm 434.9 \mathrm{ng} / \mathrm{g}$. 残留水平最高的是 DDTs $(138.8 \pm 407.3 \mathrm{ng} / \mathrm{g})$, 其次是 HCHs $(15.1 \pm 10.3 \mathrm{ng} / \mathrm{g})$ 和 HCB $(9.7 \pm 15.8 \mathrm{ng} / \mathrm{g})$, 分别占 $\sum$ OCPs 的 $80.4 \% 、 8.7 \%$ 和 $5.6 \%$. $\sum$ DDTs 中, DDT $\left(\mathrm{o}, \mathrm{p}^{\prime}\right.$-DDT、p, $\mathrm{p}^{\prime}$-DDT) 含量最高 $(132.4 \mathrm{ng} / \mathrm{g})$, 其次是 DDE $\left(\mathrm{o}, \mathrm{p}^{\prime}-\mathrm{DDE}\right.$ 和 $\left.\mathrm{p}, \mathrm{p}^{\prime}-\mathrm{DDE}, 5.4 \mathrm{ng} / \mathrm{g}\right)$ 和 $\operatorname{DDD}\left(\mathrm{p}, \mathrm{p}^{\prime}-\mathrm{DDD}, 1.0 \mathrm{ng} / \mathrm{g}\right)$. 悬浮物中 DDT 占 $\sum$ DDTs 的比例达 $95.4 \%$. HCHs 中, $\alpha-\mathrm{HCH}$ 含量相对较 高, 占 $\mathrm{HCHs}$ 的比例达 $41.4 \% ; 4$ 种 $\mathrm{HCHs}$ 异构体的含量从高到低依次为: $\alpha-\mathrm{HCH}(6.3 \mathrm{ng} / \mathrm{g})>\gamma-\mathrm{HCH}(4.1$ $\mathrm{ng} / \mathrm{g})>\beta-\mathrm{HCH}(2.7 \mathrm{ng} / \mathrm{g})>\delta-\mathrm{HCH}(2.0 \mathrm{ng} / \mathrm{g})($ 表 2$)$. 
表 2 巢湖悬浮物中 OCPs 含量

Tab. 2 OCPs concentrations in SPM of Lake Chaohu

\begin{tabular}{cccc}
\hline 污染物 & 均值 \pm 标准差/ $(\mathrm{ng} / \mathrm{g})$ & 范围 $/(\mathrm{ng} / \mathrm{g})$ & 检出率/\% \\
\hline$\alpha$-HCH & $6.3 \pm 5.1$ & $0.6 \sim 22.5$ & 100 \\
$\beta$-HCH & $2.7 \pm 3.3$ & $0.5 \sim 17.4$ & 100 \\
$\gamma$-HCH & $4.1 \pm 3.1$ & $0.7 \sim 15.8$ & 100 \\
$\delta$-HCH & $2.0 \pm 1.4$ & $0 \sim 6.3$ & 95.8 \\
$\sum$ HCHs & $15.1 \pm 10.3$ & $2.6 \sim 50.6$ & - \\
o,p'-DDE & $1.3 \pm 1.4$ & $0 \sim 5.0$ & 87.5 \\
$\mathrm{p}, \mathrm{p}^{\prime}$-DDE & $4.1 \pm 4.2$ & $0.9 \sim 20.6$ & 100 \\
$\mathrm{p}, \mathrm{p}^{\prime}$-DDD & $1.0 \pm 1.4$ & $0 \sim 5.6$ & 62.5 \\
$\mathrm{o}, \mathrm{p}$-DDT & $12.2 \pm 13.5$ & $0.5 \sim 44.2$ & 100 \\
$\mathrm{p}, \mathrm{p}^{\prime}$-DDT & $120.2 \pm 397.8$ & $0 \sim 1975.1$ & 87.5 \\
$\sum$ DDTs & $138.8 \pm 407.3$ & $3.1 \sim 2033.8$ & - \\
HCB & $9.7 \pm 15.8$ & $2.3 \sim 80.9$ & 100 \\
Heptachlor & $1.9 \pm 2.1$ & $0 \sim 9.5$ & 95.8 \\
Aldrin & $1.2 \pm 1.6$ & $0 \sim 6.4$ & 66.7 \\
Isodrin & $2.2 \pm 2.1$ & $0.5 \sim 9.5$ & 100 \\
$\gamma$-chlordane & $1.6 \pm 1.5$ & $0 \sim 7.0$ & 95.8 \\
$\alpha$-chlordane & $0.9 \pm 1.3$ & $0.0 \sim 5.4$ & 66.7 \\
Endosulfan I & $1.4 \pm 1.4$ & $0 \sim 5.8$ & 91.7 \\
Mirex & $0.1 \pm 0.3$ & $0 \sim 1.3$ & 20.8 \\
$\sum$ OCPs & $172.7 \pm 434.9$ & $18.4 \sim 2193.9$ & - \\
\hline
\end{tabular}

巢湖悬浮物中 $\mathrm{HCHs}$ 和 DDTs 与其他相 关研究结果的比较见表 3. 巢湖悬浮物中 $\mathrm{HCHs}$ 与长江南京段 ${ }^{[19]}$ 、天津大排污河 $(2007$ 年 10 月 $)^{[20]}$ 以及长江河口 ${ }^{[11,18]}$ 的测定数据 相当, 低于齐维晓等 ${ }^{[20]}$ 研究的天津北排污河 和大沽排污河 (2008 年 1 月) 悬浮物中 $\mathrm{HCH}$, 最大相差一个数量级. DDTs 污染相对严重, 高于长江南京段 ${ }^{[19]}$ 、长江口 ${ }^{[11,18]}$ 以及天津 北排污河 ${ }^{[20]}$, 最大相差一个数量级; 与天 津大沽排污河相当; 但仍低于 Tao 等 ${ }^{[21]}$ 研 究的天津 6 条河流和天津永定新河 ${ }^{[20]}$, 最 大相差一个数量级. 不同采样点之间的含 量差异可能与采样季节及采样点设置上的 差异有关.

\section{2 悬浮物中 OCPs 的时空分布}

由于数据缺失, 造成数据不配套, 本研究 用 4 个样点共有的 2010 年 9-11 月的监测 数据(表 4) 分析悬浮物中 OCPs 的空间分布 特征.

$\sum$ OCPs 在东部湖区 (JC 样点)、中部湖 区 (ZM 和 MS 样点) 和西部湖区 (TX 样点) 悬 浮物中的平均含量分别为 $46.1 \pm 21.0 、 31.0 \pm$

10.8 和 $43.1 \pm 20.9 \mathrm{ng} / \mathrm{g}$, 与 $\mathrm{Liu}$ 等研究得到的同时期水体 OCPs 含量的空间分布趋势 (中部湖区 $>$ 西部 湖区 $>$ 东部湖区) 相反 ${ }^{[22]}$. 原因可能是水体与悬浮物中 OCPs 的主要来源不同, 水体中 OCPs 可能主要来自 城市生活与工业生产废水, 流经合肥的南淝河从巢湖的西北方向不断地向湖泊注人大量污染物; 而悬浮物 中的 OCPs 可能还来自于农业生产造成的被 OCPs 污染的颗粒物. 此外, 大气的干湿沉降可能是悬浮物中 OCPs 的另一个重要来源, 特别是大气中颗粒态 OCPs 可能会对巢湖悬浮物中 OCPs 带来一定影响. Ouyang 等 ${ }^{[23]}$ 研究表明, 布设于巢湖环境保护局采样点的大气中颗粒态 OCPs 含量高于姥山采样点的大气中颗粒态 OCPs 含量, 这可能与城市中生活和工业排放有关, 而本研究中的东部水源区的 JC 样点和西部湖区的 TX 样 点分别靠近巢湖和合肥市区, 而中部湖区的 ZM 和 MS 样点位于巢湖湖区的中部, 受到城市的影响相对较 小. 此外, OCPs 还可通过沉积物的再悬浮作用重新进人悬浮物中, JC 和 TX 样点分别靠近柘臬河和十五里 河河口, 它们所受的水动力强度较湖区中部的 ZM 和 MS 样点更大, 沉积物再悬浮作用更强.

水体与悬浮物中主要 OCPs 均为 HCHs、DDTs 和 HCB. 悬浮物中 HCHs 和 HCB 含量均表现为西部湖区 最高、东部湖区次之、中部湖区最低, 而悬浮物中 DDTs 平均含量则表现为东部湖区最高、西部湖区次之、中 部湖区次之; 这也与水体中 HCHs 和 DDTs 的空间分布规律一致 ${ }^{[22,24]}$. 推测巢湖悬浮物中 DDTs 的空间分布 特征与渔船分布和船用油漆的使用有关; 巢湖约有 3500 只渔船, 其中约 $70 \%$ 分布于东部湖区, 特别是双桥 河、柘臬河以及巢湖市区的码头区域. 悬浮物中 HCHs 在西部湖区较高的原因可能为:巢湖流域农田主要分 布在湖泊西部并且西部河流人湖水量占全部河流人湖水量的 $60 \%{ }^{[24]}$.

由于巢湖西部 TX 监测点的数据不完全, 本文仅以东部的 JC 监测点以及中部的 MS 和 ZM 监测点的 数据为基础, 分析巢湖中部和东部悬浮物中 $\sum$ OCPs 和 3 种主要 OCPs (HCHs、DDTs 和 HCB) 含量的时间 变化特征( 图 2). 中部和东部湖区 $\sum$ OCPs 以及 HCHs、DDTs 和 HCB 的峰值均出现在 8 月, $5-7$ 月的 $\sum$ OCPs、DDTs 和 HCB 含量高于 9-11 月份, 而 HCHs 的时间分布则相反. 推测, 8 月份 $\sum$ OCPs 以及 HCHs、DDTs 和 HCB 含量的峰值可能与降雨径流造成颗粒物增加以及藻类暴发造成颗粒物有机碳含量增 加有关. 
表 3 巢湖与国内其它水体悬浮物中的 HCHs 和 DDTs 含量的比较

Tab. 3 Concentrations of HCHs and DDTs in SPM from lakes, rivers and estuaries in China

\begin{tabular}{|c|c|c|c|c|}
\hline 水体 & & 采样时间 & $\sum \mathrm{HCH} /(\mathrm{ng} / \mathrm{g})$ & $\sum D_{D T s} /(n g / g)$ \\
\hline 巢湖(本研究) & & 2010 年 5 月一 2011 年 4 月 & $15.1 \pm 10.3$ & $138.8 \pm 407.3$ \\
\hline 天津水体 ${ }^{[21]}$ & & 2002 年夏季 & - & $2700 \pm 1900$ \\
\hline 长江口 ${ }^{[11]}$ & & 2002 年 8 月 & $6.2 \sim 14.8$ & $3.4 \sim 25.7$ \\
\hline 长江口南岸 [18] & & 2003 年 8 月 & $6.2 \sim 14.8$ & $3.4 \sim 25.7$ \\
\hline \multirow[t]{4}{*}{ 长江南京段 ${ }^{[19]}$} & 上新河 & 1998 年 5 月 & $11.3 \pm 3.0$ & $17.7 \pm 3.6$ \\
\hline & 栖霞 & & $12.3 \pm 4.3$ & $20.7 \pm 5.5$ \\
\hline & 三江口 & & $17.4 \pm 3.2$ & $15.3 \pm 0.9$ \\
\hline & 谏壁 & & $21.9 \pm 10.7$ & $14.6 \pm 1.6$ \\
\hline \multirow[t]{12}{*}{ 天津 $^{[20]}$} & 永定新河 & 2007 年 10 月 & $13.8 \sim 53.8$ & $9.3 \sim 3565.0$ \\
\hline & & & $25.1 \pm 12.1$ & $539.4 \pm 1041.4$ \\
\hline & 北排污河 & & $25.6 \sim 42.1$ & $25.1 \sim 79.2$ \\
\hline & & & $33.8 \pm 11.6$ & $48.2 \pm 22.0$ \\
\hline & 大沽排污河 & & $5.0 \sim 38.1$ & $12.2 \sim 241.0$ \\
\hline & & & $20.9 \pm 16.5$ & $92.2 \pm 86.2$ \\
\hline & 永定新河 & 2008 年 1 月 & $2.1 \sim 68.4$ & $10.4 \sim 2476.6$ \\
\hline & & & $39.2 \pm 25.2$ & $706 \pm 995.4$ \\
\hline & 北排污河 & & 152.5 & $52.8 \sim 59.2$ \\
\hline & & & - & $55.6 \pm 3.2$ \\
\hline & 大沽排污河 & & $67.6 \sim 191.8$ & $15.4 \sim 394.4$ \\
\hline & & & $113.0 \pm 68.6$ & $187.0 \pm 143.5$ \\
\hline
\end{tabular}

表 42010 年 $9-11$ 月巢湖 4 个样点的主要 OCPs 残留水平 $(\mathrm{ng} / \mathrm{g})$

Tab. 4 Residual levels of major OCPs in SPM at four sampling sites of Lake Chaohu from September to November of 2010

\begin{tabular}{ccccc}
\hline 污染物 & JC & MS & ZM & TX \\
\hline HCHs & $18.0 \pm 8.8$ & $12.9 \pm 3.6$ & $14.5 \pm 3.4$ & $20.4 \pm 14.8$ \\
DDTs & $18.3 \pm 9.8$ & $9.7 \pm 8.4$ & $11.1 \pm 7.6$ & $12.0 \pm 5.9$ \\
HCB & $4.4 \pm 2.6$ & $2.9 \pm 1.3$ & $3.0 \pm 0.9$ & $5.1 \pm 2.8$ \\
Heptachlor & $1.8 \pm 1.2$ & $0.7 \pm 1.4$ & $1.1 \pm 0.9$ & $0.6 \pm 0.9$ \\
Aldrin & $0.2 \pm 0.3$ & - & $0.3 \pm 0.3$ & $0.1 \pm 0.3$ \\
Isodrin & $1.3 \pm 0.4$ & $0.8 \pm 0.7$ & $1.1 \pm 0.4$ & $3.0 \pm 2.5$ \\
Chlordane & $1.3 \pm 0.5$ & $1.3 \pm 1.1$ & $1.0 \pm 0.7$ & $1.2 \pm 0.7$ \\
Endosulfan I & $0.8 \pm 0.2$ & $0.4 \pm 0.4$ & $0.8 \pm 0.2$ & $0.6 \pm 0.4$ \\
Mirex & - & - & - & $0.1 \pm 0.1$ \\
¿OCPs & $46.1 \pm 21.0$ & $28.7 \pm 12.6$ & $32.8 \pm 9.9$ & $43.1 \pm 20.9$ \\
\hline
\end{tabular}

\section{3 悬浮物中 OCPs 的组成与来源解析}

2.3.1 悬浮物中 OCPs 的组成 从巢湖悬浮物中各 OCPs 污染物在不同季节占 $\sum$ OCPs 的比例可以看出, $\sum$ DDTs在 $\sum$ OCPs 中占有绝对优势, 其比例夏季最高, 为 $87.9 \%$, 而秋季最低, 为 $33.3 \%$; HCHs 仅在秋季为 主要污染物, 占 $43.9 \%$, 春、夏季分别占 $9.0 \%$ 和 $4.1 \%$; HCB 在秋季相对含量也有一定增长, 达到 $10.5 \%$; 其 余各污染物在各季节的比例均低于 $10 \%$ （图 3).

3 个季节中, DDTs 和 HCHs 在 $\Sigma$ OCPs 中均占绝对优势, 因而针对 HCHs 和 DDTs 的异构体在悬浮物中 的比例做进一步分析. HCHs 方面, 除了秋季 $\alpha-\mathrm{HCH}$ 占有绝对优势 $(56.6 \%) 、 \beta-\mathrm{HCH}$ 相对含量偏低 
东部湖区
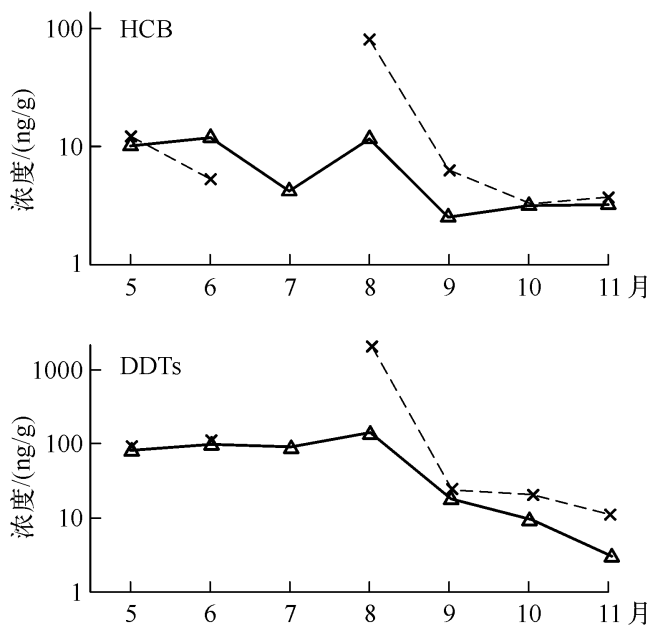
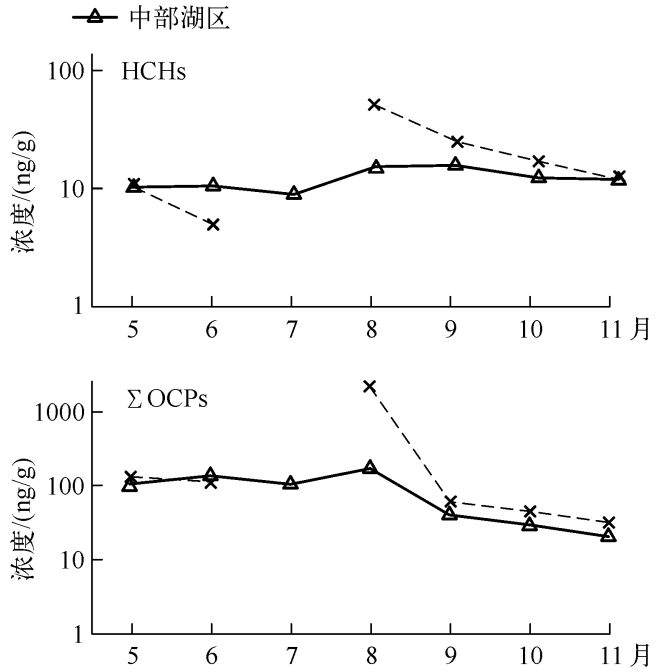

图 22010 年 5-11 月巢湖东部与中部湖区县浮物中 $\sum$ OCPs 及 3 种主要污染物 $(\mathrm{HCHs}$ 、DDTs 和 HCB) 含量的时间变化趋势

Fig. 2 Temporal trends of the total OCPs and the three major components (HCHs, DDTs and HCB) of SPM in the eastern and central areas of Lake Chaohu from May to November of 2010

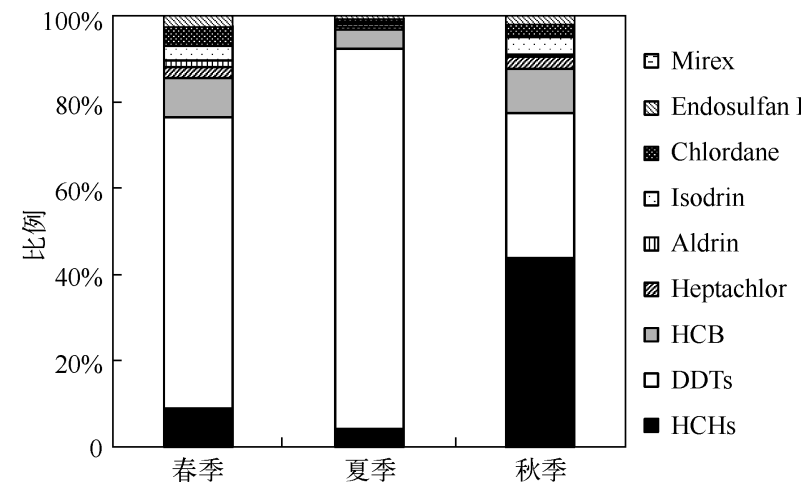

图 3 巢湖悬浮物中 OCPs 各组成占比

Fig. 3 Percentage composition of OCPs in SPM of Lake Chaohu
$(12.2 \%)$ 之外, $\alpha-\mathrm{HCH} 、 \beta-\mathrm{HCH}$ 和 $\gamma-\mathrm{HCH}$ 在其它季节所占比例相近, 3 种异构体所 占比例均在 $20 \% \sim 35 \%$ 之间; $\delta-\mathrm{HCH}$ 相对 含量在 3 个季节中均最低, 占 $\mathrm{HCHs}$ 比例 在 $11.5 \% \sim 16.3 \%$ 之间 (图 4a). DDTs 方 面, 除 $o, p^{\prime}-$ DDD 外的其余 DDTs 异构体均 在悬浮物样品中检出. 其中, DDT 在各季节 均为主要污染物, 并以 $p, p^{\prime}$-DDT 为主, $p$, $\mathrm{p}^{\prime}$-DDT 占比在 $44.6 \% \sim 91.4 \%$ 之间, o, $\mathrm{p}^{\prime}$ DDT 在春、秋两季占比也分别达到 $35.4 \%$ 和 $29.4 \%$; 其余各单体除 $\mathrm{p}, \mathrm{p}^{\prime}-\mathrm{DDE}$ 在秋 季占比略高 $(17.1 \%)$ 之外, 在其它季节比 例均未超过 $10 \%$ (图 4b).

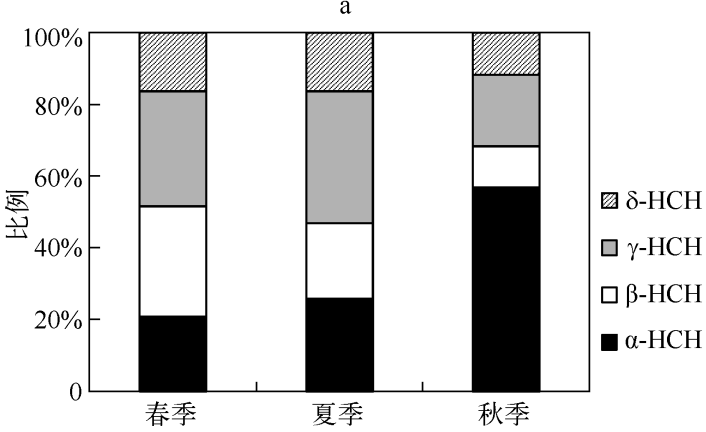

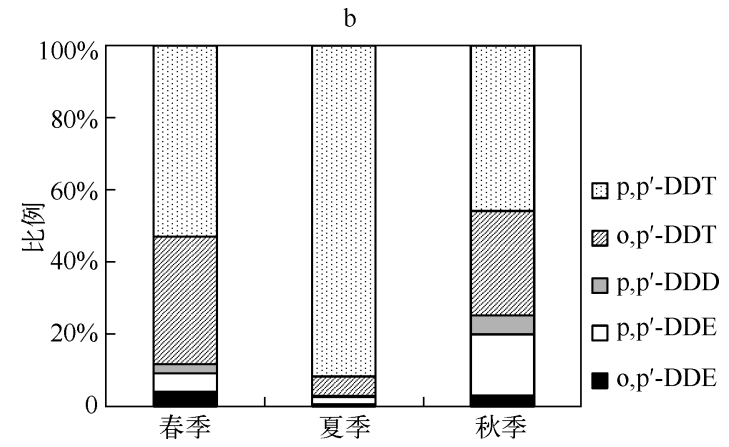

图 4 巢湖悬浮物中 $\operatorname{HCHs}(\mathrm{a})$ 和 $\operatorname{DDTs}_{\mathrm{s}}(\mathrm{b})$ 组成的季节变化

Fig. 4 Seasonal changes in the composition of HCHs (a) and DDTs (b) in SPM of Lake Chaohu 
2.3.2 悬浮物中 OCPs 的来源解析 HCHs: 环境中残留的 HCHs 可能来源于工业 HCHs 或林丹 (Lindane) 的 早期使用或最近的输人,可根据其组成的比例关系进行识别 ${ }^{[2,22-23,25-30]}$. 如果 $\alpha-/ \gamma-\mathrm{HCH}$ 比值大于 $7, \mathrm{HCHs}$ 可能来源于大气输人 ${ }^{[25-26]}$; 如果 $\alpha-/ \gamma-\mathrm{HCH}$ 比值在 $4 \sim 7$ 之间, HCHs 可能来源于工业产品; 如果 $\alpha-/ \gamma$-HCH 比值小于 4 ,则 Lindane 为其主要来源 ${ }^{[27]}$. 较高的 $\beta-/(\alpha+\gamma)$ - HCH 比值表示来源于历史上使用的工业 $\mathrm{HCHs}$ 或 Lindane ${ }^{[2]}$. 然而, 还没有公认的 $\beta-/(\alpha+\gamma)-\mathrm{HCH}$ 比值阈值来说明是历史使用或近期输人; 但 $\beta-/$ $(\alpha+\gamma)-\mathrm{HCH}<0.5$, 则认为有新的 Lindane 使用或有大气源输人 ${ }^{[23,28-30]}$. 根据以上分析, 可以构建 $\alpha-/$ $\gamma$-HCH与 $\beta-/(\alpha+\gamma)-\mathrm{HCH}$ 坐标图进行 HCH 源解析 ${ }^{[22-23,29-30]}$.

巢湖水体悬浮物中 $\alpha-/ \gamma-\mathrm{HCH}$ 与 $\beta-/(\alpha+\gamma)-\mathrm{HCH}$ 关系如图 5 所示. $\alpha-/ \gamma$-HCH 比值在 $0.38 \sim 3.30$ 之 间,除 5 月的 JC、MS 样品, 7 月的 ZM 样品, 8 月的 JC 样品外, 大部分悬浮物样品 $\beta-/(\alpha+\gamma)-\mathrm{HCH}<0.5$. 这 些特征表明,巢湖悬浮物中的 HCHs 主要来源于近期 Lindane 的使用.

DDTs: 分析环境中 DDTs 的组成可判断 其来源 ${ }^{[31-35]}$. 环境中高比值的 $o, p^{\prime}-/ p, p^{\prime}-$ DDT 一般认为是受到三氯杀螨醇 (Dicofol) 的 污染, 而比值在 0.2 左右则主要是工业 DDT 的使用 ${ }^{[32]}$. DDT/ (DDE + DDD ) $\geqslant 1$ 表示有 新的 DDT 输人,DDT/ (DDE + DDD ) $<1$ 表示 历史输人 ${ }^{[34]} . \mathrm{DDD} / \mathrm{DDE}>1$, 表示代谢环境 主要为厌氧条件, $\mathrm{DDD} / \mathrm{DDE}<1$, 表示代谢环 境主要为好氧条件 ${ }^{[31,33]}$. 根据上述分析, 可 以构建 $\mathrm{o}, \mathrm{p}^{\prime}-/ \mathrm{p}, \mathrm{p}^{\prime}$-DDT与 DDT/( DDE + DDD) 的坐标图解析 DDTs 来源与施用历 史 $^{[22-23,31]}$, 也可以利用 DDTs 组成三角图来分 析 DDTs 的施用历史及其代谢环境 ${ }^{[35]}$.

对悬浮物中 DDTs 来源进行解析 (图 6) 发现, 除 10 月的 MS 样点、11月的 ZM 和 TX 3 个样品中未检出 $\mathrm{p}, \mathrm{p}^{\prime}$-DDT 之外, 其余各样

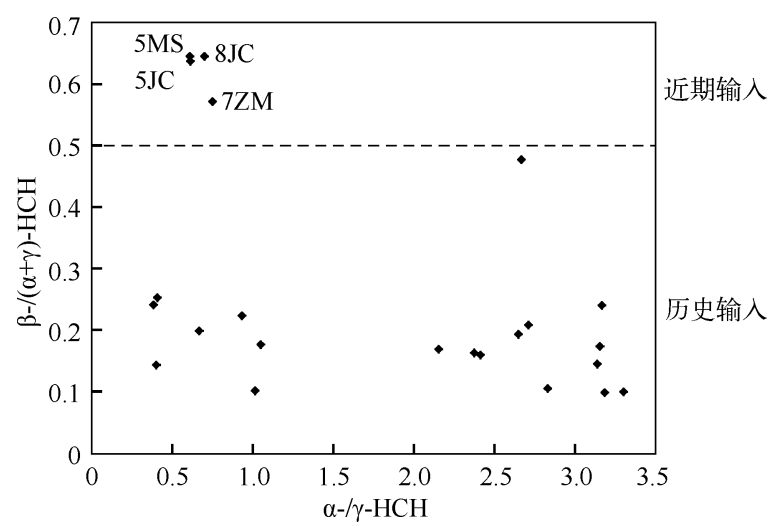

图 5 2010 年 5-11 月巢湖悬浮物中 $\mathrm{HCHs}$ 来源解析 (样点号前的数字代表采样月份,下同)

Fig. 5 Source apportionment of HCHs in the SPM of Lake Chaohu from May to November of 2010 点 $0, p^{\prime}-/ p, p^{\prime}-D D T$ 比值在 $0.02 \sim 0.98$ 之间, 均小于 1 , 表明悬浮物中监测到的 DDTs 主要是残留在环境中的 工业 DDTs. 各样点各月份样品中 DDT/ (DDD + DDE) 的比值范围在 1.86 98.72 之间, 比值均大于 1 , 比值 最高的样品为 8 月的 JC 样品. 这说明悬浮物中可能存在新的 DDTs 输人. 但考虑到对巢湖水体 ${ }^{[22]}$ 和大 气 ${ }^{[23]}$ 的研究中均未发现这一情况,故推测 DDTs 不是来源于污染物向水体的直接排放,而可能是周边土壤 中肥料的施用或含有 DDTs 的生活、工业垃圾废渣等颗粒物随地表径流进人湖体. 从降解环境的角度,绝大 多数样品中都以 DDE 为主要代谢产物, 说明 DDTs 在悬浮颗粒物上的降解以有氧条件为主.
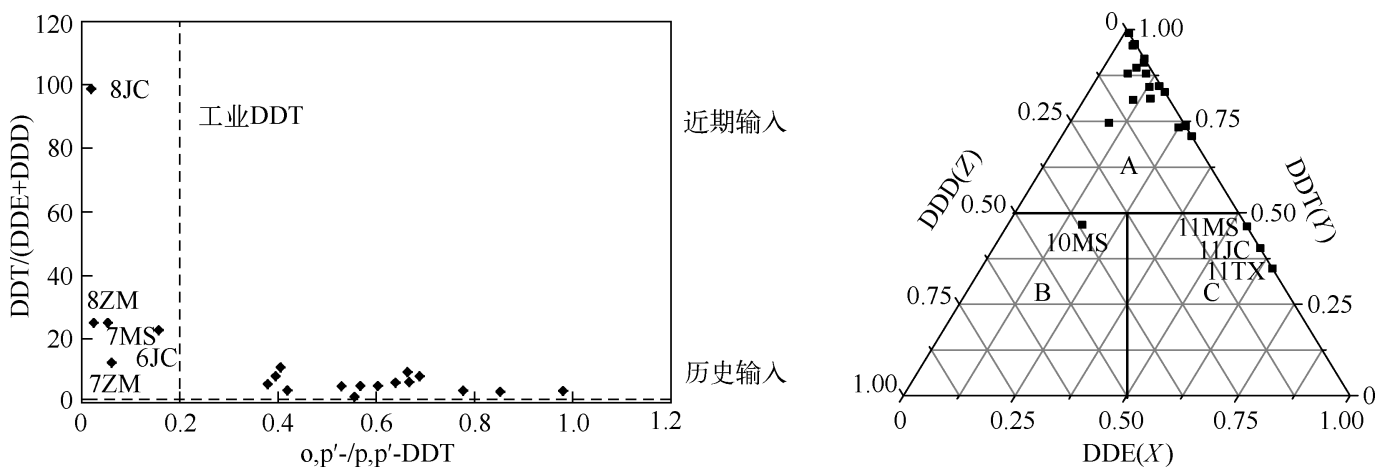

图 62010 年 5-11 月巢湖悬浮物中 DDTs 来源解析

Fig. 6 Source apportionment of DDTs in the SPM of Lake Chaohu from May to November of 2010 
Chlordane: Chlordane 有反式与顺式两种同分异构体, 在环境中反式氯丹 ( $\gamma$-Chlordane) 要比顺式氯丹 $(\alpha-$ Chlordane) 易于降解 ${ }^{[36]}$; 所以, $\alpha-/ \gamma$-Chlordane 的比值可以应用于判断是否有近期污染引人. 工业氯丹中 $\alpha-/$ $\gamma$-Chlordane 的比值约为 $0.77^{[37]}$, 当 $\alpha-/ \gamma$-Chlordane $<0.77$ 时, 表明有新的工业 Chlordane 输人. 如果在环境 介质中, $\alpha-/ \gamma$-Chlordane 的比值大于 1 , 表明环境中无新的 Chlordane 输人 ${ }^{[38]}$. 本研究中大多数样品的 $\alpha-/ \gamma-$ Chlordane $<0.77$ (图 7), 可以推断出巢湖区域存在新的工业 Chlordane 的输人. 这与大气 Chlordane 源解析 结果一致 ${ }^{[23]}$.

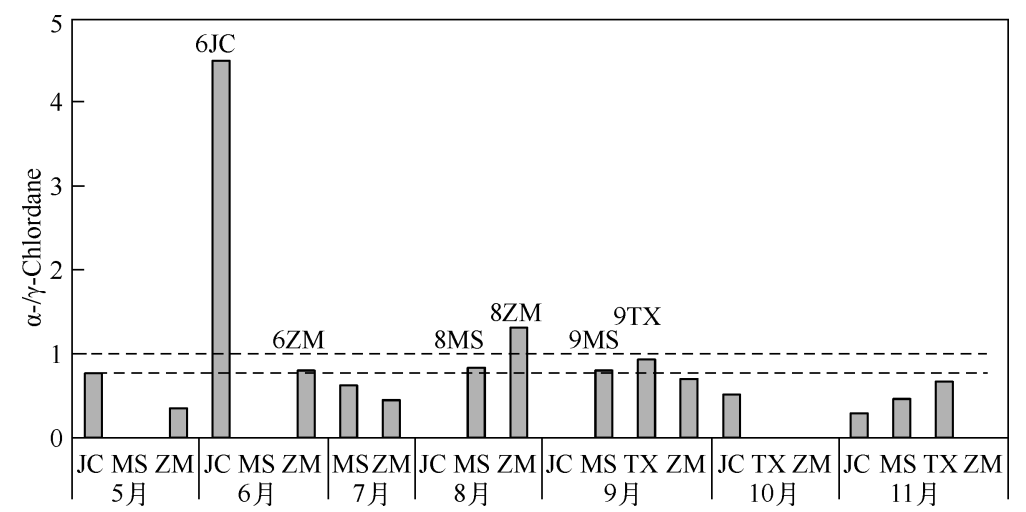

图 72010 年 5-11 月巢湖悬浮物中氯丹来源解析

Fig. 7 Source apportionment of chlordane in the SPM of Lake Chaohu from May to November of 2010
$\mathrm{HCB}$ : HCB 主要作为生 产五氯酚 (PCP) 和五氯酚 钠 ( PCP-Na) 的中间体. PCP 可用于木材防腐和除 草杀虫剂; 而 PCP-Na 在我 国一些地区用于防治血吸 虫, 曾经在血吸虫病流行地 区被广泛用来杀灭钉螺. 1980s 起 PCP 已被禁止生 产和使用, 但 PCP-Na 因为 价格便宜、使用方便、灭血 吸虫病的作用无法取代使 得其仍在我国有一定量的 使用. 此外, HCB 不仅作为

一种污染物存在于这些产品中,还可来自于一些含氯化学工业或含氯废物的焚烧.

巢湖流域是湖沼型为主的血吸虫病流行区, 历史累计有螺面积 $1.2 \times 10^{8} \mathrm{~m}^{2}$, 曾有 4 万余名血吸虫病患 者 $^{[23]}$. 推测巢湖悬浮物中含有较高的 HCB 与本地区使用 PCP-Na 防治血吸虫病以及工 (含氯化学工业)、农 ( 作为杀虫剂使用) 业生产等的排放有关.

\section{4 潜在生态风险评价}

由于对水体悬浮物中污染物进行生态风险评价的文献较少, 目前, 尚没有统一的方法与标准进行悬浮 物中微量有机污染物的生态风险评价. 有研究表明, 沉积物生态风险评价的方法与标准可以用于悬浮物生 态风险评价 ${ }^{[10,39]}$. 目前, 已经有很多学者进行了 OCPs 沉积物质量标准( sediment quality guidelines, SQGs) 的 研究 ${ }^{[40-42]}$. 本文利用 MacDonald 等于 2000 年提出的淡水环境中的共识沉积物质量基准 (consensus-based sediment quality guidelines, CB-SQGs $)^{[42]}$, 对巢湖悬浮物中 OCPs 的潜在生态风险进行了评价. 该基准选用 阈值效应含量 (consensus-based concentration, TECs) 和可能效应含量 (consensus-based probable effect concentration, PECs) 来评估污染物的生态风险. 污染物含量低于 TECs 指示不会对水生生物产生负面影响; 污染物 含量高于 PECs 指示通常会对水生生物产生负面影响; 介于两者之间则指示可能会对水生生物产生负面 影响 $^{[42]}$.

运用此基准对巢湖悬浮物中 OCPs 的生态风险进行评价, 结果见表 5. 巢湖悬浮物中 Chlordane、DDD、 Heptachlor 含量低于 TECs, 不会对水生生物产生不利影响; 然而 DDT ( p, p'-DDT 和 o, p'-DDT) 含量高于 PECs, 会对水生生物产生潜在的不利影响. 研究中污染物含量高于 PECs 的情况, 从季节上看主要发生在夏 季, 而从空间分布角度看主要发生在 ZM 和 MS 样点. 总之, 巢湖悬浮物中 Lindane、DDTs 的残留可能对巢湖 水生态环境造成一定危害, 应加以关注.

\section{3 结论}

1）巢湖悬浮物中的 $\sum$ OCPs 年平均浓度为 $172.7 \pm 434.9 \mathrm{ng} / \mathrm{g}, \sum \mathrm{HCHs}$ 年均浓度为 $15.1 \pm 10.3 \mathrm{ng} / \mathrm{g}$, $\sum$ DDTs 年均浓度为 $138.8 \pm 407.3 \mathrm{ng} / \mathrm{g}$. DDTs 在夏季污染水平达到最高. 空间污染程度由重到轻依次为东 部湖区及水源区 $>$ 西部湖区 $>$ 中部湖区. 
表 5 巢湖悬浮物中 OCPs 含量水平与 OCPs 沉积物质量标准的比较

Tab. 5 Comparison between OCPs levels in this study and CB-SQGs values

\begin{tabular}{cccc}
\hline OCPs & 巢湖悬浮物中 OCPs 含量 $/(\mathrm{ng} / \mathrm{g}(\mathrm{dw}))$ & $\mathrm{TECs} /(\mathrm{ng} / \mathrm{g}(\mathrm{dw}))$ & $\mathrm{PECs} /(\mathrm{ng} / \mathrm{g}(\mathrm{dw}))$ \\
\hline Chlordane & $2.45(0 \sim 15.77)$ & 3.24 & 17.6 \\
DDD & $0.98(0 \sim 11.23)$ & 4.88 & 28.0 \\
DDE & $5.45(1.01 \sim 25.56)$ & 3.16 & 31.3 \\
DDT & $137.18(0 \sim 3343.95)$ & 4.16 & 62.9 \\
EDDTs & $143.60(1.73 \sim 3369.43)$ & 5.28 & 572 \\
Heptachlor & $1.80(0 \sim 12.74)$ & 2.47 & 16.0 \\
Lindane & $4.24(0.62 \sim 19.11)$ & 2.37 & 4.99 \\
\hline
\end{tabular}

2) 巢湖悬浮物中, DDTs 在 $\Sigma$ OCPs 中占有绝对优势, 其比例夏季最高而秋季最低; HCHs 仅在秋季为主 要污染物; $\mathrm{HCB}$ 在秋季相对浓度也有一定增长;其余各污染物在各季节的比例均很低.

3) 巢湖悬浮物中的 HCHs 主要来源于 Lindane 近期非法使用. 而DDTs 主要来源于工业 DDTs 的使用, 在春季可能存在 DDTs 的非法使用;悬浮物中 DDTs 的降解环境主要为有氧环境.

4) 巢湖悬浮物中 $\mathrm{p}, \mathrm{p}^{\prime}$-DDT 和 o, $\mathrm{p}^{\prime}$-DDT 会对水生生物产生潜在的不利影响.

\section{4 参考文献}

[ 1 ] Jones KC, de Voogt P. Persistent organic pollutants (POPs) : state of the science. Environmental Pollution, 1999, 100 : 209-221.

[ 2 ] Willett KL, Ulrich EM, Hites RA. Differential toxicity and environmental fates of hexachlorocyclohexane isomers. Environmental Science and Technology, 1998, 32(15):2197-2207.

[ 3 ] Quan X, Niu JF, Chen Set al. Effects of $\mathrm{Fe}_{2} \mathrm{O}_{3}$, organic matter and carbonate on photocatalytic degradation of lindane in the sediment from the Liao River, China. Chemosphere, 2003, 52:1749-1755.

[ 4 ] Wong MH, Leung AOW, Chan JKY et al. A review on the usage of POP pesticides in China, with emphasis on DDT loadings in human milk. Chemosphere, $2005,60: 740-752$.

[ 5 ] Tang ZW, Yang ZF, Shen ZY et al. Residues of organochlorine pesticides in water and suspended particulate matter from the Yangze River catchment of Wuhan, China. Environmental Monitoring and Assessment, 2008, 137:427-439.

[ 6 ] Tao S, Xu FL, Wang XJ et al. Organochlorine pesticides in agricultural soil and vegetables from Tianjin, China. Environmental Science and Technology, 2005, 39(8) :2494-2499.

[ 7 ] Tao S, Liu WX, Li Y et al. Organochlorine pesticides contaminated surface soil as reemission source in the Haihe Plain, China. Environmental Science and Technology, 2008, 42(22):8395-8400.

[ 8 ] Gewurtz SB, Helm PA, Waltho J et al. Spatial distributions and temporal trends in sediment contamination in Lake St. Clair. Journal of Great Lakes Research, 2007, 33(3) :668-685.

[ 9 ] Li J, Zhang G, Guo LL et al. Organochlorine pesticides in the atmosphere of Guangzhou and Hongkong: Regional sources and long-range atmospheric transport. Atmospheric Environment, 2007, 41:3889-3903.

[10] Feng JL, Zhai MX, Liu Q et al. Residues of organochlorine pesticides (OCPs) in upper reach of the Huaihe River, East China. Ecotoxicology and Environmental Safety, 2011, 74:2252-2259.

[11] Liu M, Cheng SB, Ou DN et al. Organochlorine pesticides in surface sediments and suspended particulate matters from the Yangtze estuary, China. Environmental Pollution, 2008, 156:168-173.

[12] Dai GH, Liu XH, Liang G et al. Distribution of organochlorine pesticides (OCPs) and polychlorinated biphenyls (PCBs) in surface water and sediments from Baiyangdian Lake in North China. Journal of Environmental Sciences, 2011, 23(10) : 1640-1649.

[13] Yang RQ, Lv AH, Shi JB et al. The levels and distribution of organochlorine pesticides (OCPs) in sediments from the Haihe River, China. Chemosphere, 2005, 61:347-354.

[14] Doong RA, Lee SH, Lee CC et al. Characterization and composition of heavy metals and persistent organic pollutants in water and estuarine sediments from Gaoping River, Taiwan. Marine Pollution Bulletin, 2008, 57 :846-857.

[15 ] Zhao ZH, Zhang L, Wu JL et al. Assessment of the potential mutagenicity of organochlorine pesticides (OCPs) in contaminated sediments from Taihu Lake, China. Mutation Research, 2010, 696:62-68.

[16] Barakat AO, Mostafa A, Wade TL et al. Spatial distribution and temporal trends of persistent organochlorine pollutants in sediments from Lake Maryut, Alexandria, Egypt. Marine Pollution Bulletin, 2012, 64:395-404. 
[17] Barakat AO, Mostafa A, Wade TL et al. Assessment of persistent organochlorine pollutants in sediments from Lake Manzala, Egypt. Marine Pollution Bulletin, 2012, 64:1713-1720.

[18] 刘华林, 刘 敏, 程书波等. 长江口南岸水体 SPM 和表层沉积物中 OCPs 的赋存. 中国环境科学, 2005, 25(5): $622-626$.

[19] 蒋 新, 许士奋, Martens D 等. 长江南京段水、悬浮物及沉积物中多氯有毒有机污染物. 中国环境科学, 2000,20 (3) : 193-197.

[20] 齐维晓, 刘会娟, 曲久辉等. 天津主要纳污及人海河流中有机氯农药的污染现状及特征. 环境科学学报, 2010, 30 ( 8 ) : 1543-1550.

[21] Tao S, Li BG, He XC et al. Spatial and temporal variations and possible sources of dichlorodiphenyltrichloroethane (DDT) and its metabolites in rivers in Tianjin, China. Chemosphere, 2007, 68:10-16.

[22] Liu WX, He W, Qin N et al. Residues, distributions, sources and ecological risks of OCPs in the water from Lake Chao, China. The Scientific World Journal, 2012. doi:10.1100/2012/897697.

[23] Ouyang HL, He W, Qin N et al. Levels, temporal-spatial variations and sources of organochlorine pesticides in ambient air of Lake Chaohu, China. The Scientific World Journal, 2012. doi:10. 1100/2012/504576.

[24] He W, Qin N, He QS et al. Characterization, ecological and health risks of DDTs and HCHs in water from a large shallow Chinese lake. Ecological Informatics, 2012, 12:77-84.

[25] Iwata H, Tanabe S, Tatsukawa R. A new view on the divergence of HCH isomer compositions in oceanic air. Marine Pollution Bulletin, 1993, 26:302-305.

[26] Iwata H, Tanabe S, Ueda K et al. Persistent organochlorine residues in air, water, sediments, and soils from the Lake Baikal region, Russia. Environmental Science and Technology, 1995, 29(3):792-801.

[27] Walker K, Vallero AD, Lewis GR. Factors influence the distribution of lindane and other hexachlorocyclohexance in the environment. Environmental Science and Technology, 1999, 33(24) :4372-4378.

[28 ] Liu WX, Li Y, Zuo Q et al. Residual characteristics of HCHs and DDTs in surface soils from the western zone of Bohai Bay. Acta Scientiae Circumstantiae, 2008, 28(1):142-149.

[29] Xu FL, Kong XZ, He W et al. Distributions, sources and ecological risks of HCHs in the sediments from Haihe Plain, Northern China. Environmental Sciences and Pollution Research, 2013, 20 (4):2009-2019.

[30] Wang Y, Wu WJ, He W et al. Residues and ecological risks of organochlorine pesticides in Lake Small Baiyangdian, North China. Environmental Monitoring and Assessment, 2013, 185 (1) :917-929.

[31] Hitch RK, Day HR. Unusual persistence of DDT in some western USA soils. Bulletin of Environmental Contamination and Toxicology, 1992, 48(2):259-264.

[32] Qiu XH, Zhu T, Yao B et al. Contribution of dicofol to the current DDT pollution in China. Environmental Science and Technology, 2005, 39:4385-4390.

[33 ] Sun YZ, Wang XT, Li XH et al. Distribution of persistent organochlorine pesticides in tissue /organ of silver carp ( Hypophthalmichthys molitrix) from Guanting Reservoir, China. Journal of Environmental Sciences, 2005, 17(5) :722-726.

[34] Pandit GG, Sahu SK, Sharma S et al. Distribution and fate of persistent organochlorine pesticides in coastal marine environment of Mumbai. Environment International, 2006, 32(2) :240-243.

[35] Wang Y, He W, Qin N et al. Distributions, sources, and ecological risks of DDT-related contaminants in water, suspended particulate matter, and sediments from Haihe Plain, Northern China. Environmental Monitoring and Assessment, $2013, \mathbf{1 8 5}(2): 1777-1790$.

[36] Eitzer BD, Mattina MJI, Iannuchi-Berger W. Compositional and chiral profiles of weathered chlordane residues in soil. Environmental Toxicology and Chemistry, 2001, 20(10):2198-2204.

[37] Sovocool GW, Lewis RG, Harless RL et al. Analysis of technical chlordane by gas chromatography/mass spectrometry. Analytical Chemistry, 1977, 49(6): :734-740.

[38 ] Bidleman TF, Jantunen LMM, Helm PA et al. Chlordane enantiomers and temporal trends of chlordane isomers in Arctic air. Environmental Science and Technology, 2002, 36(4) :539-544.

[39] Schubert B, Heininger P, Keller M et al. Monitoring of contaminants in suspended particulate matter as an alternative to sediments. Trends in Analytical Chemistry, 2012, 36:58-70.

[40] Long ER, MacDonald DD, Smith SL et al. Incidence of adverse biological effects within ranges of chemical concentrations in marine and estuarine sediments. Environmental Management, 1995, 19(1) :81-97.

[41] Smith SL, MacDonald DD, Keenleyside KA et al. A preliminary evaluation of sediment quality assessment values for freshwater ecosystems. Journal of Great Lakes Research, 1996, 22 (3) :624-638.

[42] MacDonald DD, Ingersoll CG, Berger TA. Development and evaluation of consensus-based sediment quality guidelines for freshwater ecosystems. Archives of Environment Contamination and Toxicology, 2000, 39:20-31. 